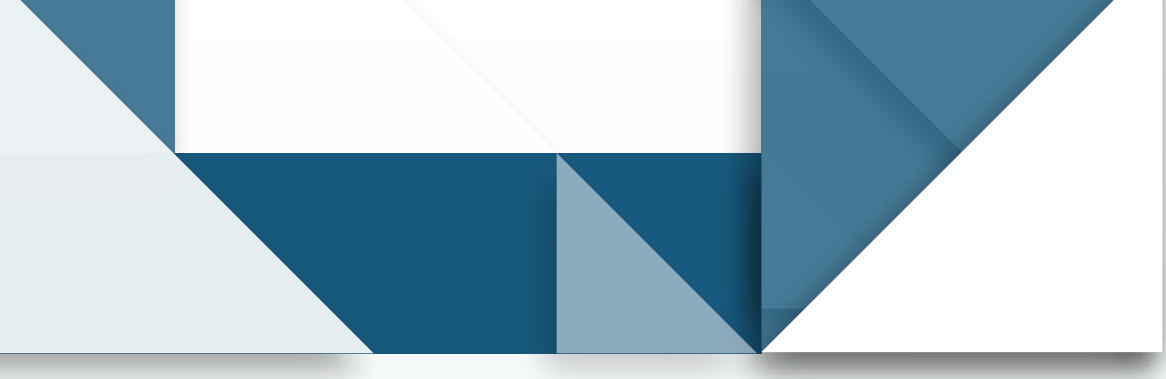

\title{
1) El pensamiento covariacional y GeoGebra: herramientas para la explicación científica de algunas realidades
}

- Covariational Thinking and GeoGebra: Tools for Scientific Explanation of Certain Realities

- O pensamento covariacional e GeoGebra: ferramentas para a explicação científica de algumas realidades

\section{Resumen}

El siguiente artículo presenta los avances del proyecto de investigación "El laboratorio de pensamiento variacional: Una experiencia para estudiantes de poblaciones vulnerables", el cual tiene como objetivo diseñar un laboratorio de ciencias en donde se analice el uso del pensamiento covariacional en prácticas experimentales que potencien el trabajo en grupo y la convivencia con estudiantes de poblaciones vulnerables. La aproximación teórica en la que se acoge el proyecto es la teoría socioepistemológica la cual es una teoría emergente en educación matemática y pertinente para las realidades de nuestra comunidad educativa. La población que se ha escogido es la institución educativa Los Pinos en el barrio Los Laches en Bogotá. Se entiende el laboratorio como un escenario que propicia el trabajo en equipo, y facilita un uso del conocimiento matemático en escenarios de experimentación física. Se pretende apoyar el trabajo experimental con el uso de herramientas tecnológicas que les permita a los estudiantes desarrollar competencias relacionadas con el aprendizaje asistido por el computador, en este caso con el software GeoGebra. El uso de este programa le dará a los estudiantes la posibilidad de analizar gráficamente la covariación de algunas magnitudes definidas por la necesidad de resolver problemas de la cotidianidad de los integrantes del laboratorio.

Palabras clave

Pensamiento variacional; GeoGebra; socioepistemología; población vulnerable

\begin{abstract}
This paper presents the progress of the research project titled "Variational thinking laboratory: An experience for students from vulnerable populations," which aims to design a science laboratory where the use of covariational thinking in experimental practices that promote group work and coexistence with students from vulnerable populations is analyzed. The theoretical approach of the project is socio-epistemological theory, which is an emerging theory in mathematics education and relevant to the realities of our educational community. The population chosen was
\end{abstract}

Carlos Eduardo León-Salinas*

Doctorando en Matemática Educativa, Universidad La Gran Colombia, Director de la Licenciatura en Matemáticas, Bogotá, Colombia. Correo electrónico: carlos.leon@ugc.edu.co Orcid: 0000-0002-5220-1635 
Los Pinos School in Los Laches neighborhood in Bogotá. The laboratory is understood as a scenario that fosters teamwork and facilitates the use of mathematical knowledge in physical experimentation scenarios. The purpose is to support experimental work with the use of technological tools that allow students to develop skills related to computer-assisted learning, in this case, the GeoGebra software. Using this program will give the students the chance to graphically analyze the covariation of some magnitudes defined by the need to solve problems of the daily life of the members of the laboratory.

Keywords

Variational thinking; GeoGebra; socio-epistemology; vulnerable population

\section{Resumo}

O seguinte artigo apresenta os avanços do projeto de pesquisa "O laboratório de pensamento variacional: uma experiência para estudantes de populações vulneráveis", que visa planejar um laboratório de ciências onde seja possível analisar o uso do pensamento covariacional em práticas experimentais que estimulam o trabalho em grupo e a convivência com estudantes de populações vulneráveis. A aproximação teórica utilizada para o projeto é a teoria socioepistemológica, que é uma teoria emergente na educação matemática e pertinente para as realidades de nossa comunidade educativa. A população escolhida é a instituição educativa Los Pinos no bairro Los Laches em Bogotá. O laboratório é concebido como um cenário que estimula o trabalho em equipe e facilita o uso do conhecimento matemático em cenários de experimentação física. Pretende-se sustentar o trabalho experimental com o uso de ferramentas tecnológicas que permitem aos estudantes desenvolver competências relacionadas com a aprendizagem assistida por computador, neste caso com o software GeoGebra. $\bigcirc$ uso deste programa daria aos estudantes a possibilidade de analisar graficamente a variação de algumas magnitudes definidas pela necessidade de resolver problemas do dia-a-dia dos membros do laboratório.

Palavras-chave

Pensamento variacional; GeoGebra; socioepistemologia; população vulnerável 


\section{Introducción}

El siguiente artículo presenta los resultados de un proyecto de investigación realizado por el programa de Licenciatura en Matemáticas y Tecnologías de la información de la Universidad La Gran Colombia en el 2016 y responde a los principios institucionales de llevar el conocimiento a distintos grupos en nuestra sociedad. En este caso, la propuesta inicial era diseñar un escenario para la construcción de conocimiento matemático destinado a estudiantes de poblaciones vulnerables que difícilmente cuentan con herramientas tecnológicas que potencien la elaboración de hipótesis, la toma de datos y la verificación de resultados. A continuación, se hará una presentación de cada uno de los elementos que conformaron los antecedentes de este proyecto.

\section{Antecedentes}

La Universidad La Gran Colombia está ubicada en el centro de Bogotá, una zona turística y con algunos rasgos coloniales que escapan al cambio normal de los tiempos modernos. A cinco minutos se encuentra Los Laches, un barrio de población vulnerable y con muchos problemas de tipo social reflejados en altos índices de inseguridad y de riñas, factores que también están presentes en sus instituciones educativas. Este barrio, inicialmente, fue una invasión, legalizada hace más de cincuenta años, pero aún hoy en día presenta un asentamiento de desplazados de la violencia y personas de bajos recursos que construyen sus viviendas en sitios aledaños a los cerros que acompañan el imponente paisaje del sector. A un lado, la inmensidad de una ciudad gobernada por sus altos edificios y sus numerosas calles; al otro lado, el verde vivo de la prominente naturaleza presente en las montañas capitalinas.

El barrio cuenta con tres instituciones educativas, las cuales son de carácter oficial y atienden a una población de más de 2.500 estudiantes entre los 5 y los 20 años de edad. Este proyecto se desarrollará en una de estas instituciones, el colegio Los pinos, el cual cuenta con unos 450 estudiantes. Los factores de violencia, riñas y desempleo mencionados anteriormente también afectan a los estudiantes. La violencia entre los estudiantes es un aspecto presente en la institución, los grados de tolerancia son mínimos y han sido numerosos los esfuerzos por mejorar el clima institucional alrededor de la solución pacífica de conflictos. Esta es una tarea que exige una participación colectiva que debe recibir aportes desde cada una de las asignaturas escolares. Por ello, uno de los principales objetivos de esta propuesta es consolidar un escenario que fomente el trabajo en grupo para lograr una construcción social del conocimiento, en este caso, de las matemáticas. Las matemáticas han sido durante mucho tiempo un sinónimo de difícil no solo en el salón de clase, sino fuera de él. En diferentes medios de comunicación, se hacen expresos testimonios de periodistas, deportistas, políticos, modelos y hasta algunos académicos según los cuales las matemáticas no han sido un conocimiento útil para sus profesiones. ¿̇ué puede pensar un estudiante acerca de aprender matemáticas?

En la actualidad, se ha ido acrecentando la preocupación por la enseñanza y aprendizaje de las matemáticas, que ha hecho que numerosas comunidades académicas fijen sus ojos y le den una merecida importancia a los proyectos de investigación en educación matemáti$\mathrm{ca}$, disciplina muy activa tanto en eventos como en publicaciones en los últimos años. La comprensión de conocimientos matemáticos se ha convertido en el santo grial de distintos actores del proceso educativo, desde el profesor de aula hasta investigadores especializados han gastado recursos en el entendimiento de cómo hacer que un individuo $A$ entienda un cierto concepto $B$ en un determinado contexto $C$, convirtiéndose 
en una ecuación que a su vez involucra un sinfín de variables que muchas veces no son tenidas en cuenta y que se limitan a las tres expuestas inicialmente.

Entre esas variables se encuentra la falta de interés no solo del estudiante, sino también del docente, por entender las matemáticas como un conocimiento que se puede usar para la interpretación de fenómenos reales. En diversas investigaciones (Godino y Batanero, 1996; Gonzales, 2005; Miranda, 2009), se ha establecido el desinterés por parte de los estudiantes de matemáticas que radica en la falta de motivación y que manifiestan con rechazo por la asignatura o las actividades relacionadas con ella. El filósofo y matemático inglés Alan Bishop, en su libro Enculturación matemática, comenta:

Las matemáticas se encuentran en una posición nada envidiable: son una de las materias escolares más importantes que los niños de hoy deben estudiar y, al mismo tiempo, una de las peor comprendidas. Su reputación intimida. Todo el mundo sabe que son importantes y que su estudio es necesario. Pero pocas personas se sienten cómodas con ellas; hasta tal punto que en muchos países es totalmente aceptable, en el ámbito social, confesar la ignorancia que se tiene de ellas, fanfarronear sobre la propia incapacidad para enfrentarse a ellas ie incluso afirmar que se les tiene fobia. (Bishop, 1999).

La hipótesis inicial de este trabajo está basada en que el interés de los estudiantes se dará a partir de contextos que propongan un uso del conocimiento matemático que resignifique ideas y conceptos que en la matemática escolar han tenido una presentación abstracta y alejada de la realidad. A pesar de situaciones y visiones experimentales que ha tenido las matemáticas a lo largo de la historia, no es frecuente apreciar esta relación en los discurso de aula, tampoco en los libros de texto y ni siquiera en los planes de estudio de las instituciones. Arrieta (2003) reporta esta dificultad al afirmar que el peso de los fenómenos físicos en la clase de matemáticas es escaso, a pesar que nociones y procedimientos matemáticos han surgido del proceso de comprender fenómenos físicos reales. Cordero y Martínez (2001) atribuyen el desconocimiento de la importancia de la física en la enseñanza de las matemáticas al privilegiar argumentos de corte analítico que toman a los conceptos matemáticos como objetos elaborados, alejados totalmente de argumentos situacionales.

El saber matemático impartido en el aula debe ser un saber vivo, que evoluciona y que busca una relación directa con saberes de otras disciplinas al formar en el conocimiento matemático un carácter social que lo convierte en una herramienta de argumentación del individuo en un contexto sociocultural determinado. Por lo tanto, la argumentación se forma en el tránsito entre las diferentes disciplinas científicas en las que se puede estudiar la generación de un conocimiento matemático, dotado de un contexto significativo y de las actividades y herramientas que permiten su construcción (Buendía, 2004). Buendía considera dos aspectos primordiales para el estudio de las matemáticas: lo que sucede en la clase de matemáticas debe estar ligado a lo que sucede en otras 
clases y a lo que sucede fuera de ellas (contexto sociocultural) y la naturaleza misma del conocimiento matemático.

\section{Marco teórico}

Específicamente en el conocimiento matemático, se trabajará la idea del pensamiento covariacional, que, según Carlson y Jacobs (2003), refiere a aquellas actividades cognitivas implicadas en la coordinación de dos cantidades que varían mientras se atiende a las formas en que cada una de ellas cambia con respecto a la otra. El pensamiento covariacional es el fundamento para construir el concepto de función y de ahí su importancia para la comprensión de los resultados más importantes del cálculo como el límite - la integral. En la estructura de los estándares curriculares, el Ministerio de Educación Nacional (MEN) define los cinco tipos de pensamiento que deben desarrollar los estudiantes a lo largo de la escuela, uno de ellos es el pensamiento variacional, el cual tiene que ver con el reconocimiento, la percepción, la identificación y la caracterización de la variación y el cambio en diferentes contextos, así como su descripción, modelación y representación en distintos sistemas o registros simbólicos (MEN, 2004).

El estudio de la variación no se limita a un curso o etapa particular y debe estar presente en los contenidos y saberes desarrollados a lo largo de todo el ciclo escolar de los estudiantes, además de relacionarse con otros tipos de pensamiento como el geométrico, el numérico o el aleatorio. Su estudio comienza a temprana edad con el estudio de frecuencias y patrones repetitivos en figuras o en relaciones numéricas que propician que el estudiante construya estrategias en torno a cómo encontrar un patrón. Otro tipo de actividades que pueden servir para evidenciar un estudio de la variación se presenta en otras áreas que pueden acompañar el proceso en matemáticas. Por ejemplo, el registro del crecimiento de una planta en la clase de ciencias es un ejercicio muy común, donde el estudiante debe registrar los cambios que va presentando la planta a lo largo de los días. Este tipo de actividades tiene una gran importancia desde los significados que le dan los estudiantes a las magnitudes que van cambiando; en este caso, el tiempo y la altura son medidas que se hacen más significativas para el estudiante debido a que él mismo las realiza y el estudio de su comportamiento tiene relación con las condiciones del experimento, como el tipo de planta o la temperatura.

Este tipo de actividades contextualiza el pensamiento variacional y demuestra su importancia en otras áreas del conocimiento que llegan a convertirse en generadoras de problemas que implican la modelación de algún fenómeno físico y donde, para la visualización de los comportamientos, se cuenta con una gran variedad de representaciones como gráficas, tablas, esquemas y hasta lo verbal, lo que implica la gran relación que existe entre el pensamiento variacional y los sistemas de representación. Los estudiantes pueden hacer una tabla, una gráfica o describir con palabras el comportamiento del crecimiento de la planta.

Además de la importancia curricular que tiene el pensamiento covariacional, es muy significativa la interpretación que se puede hacer de él en contextos no escolares, en los cuales se presenta de manera más cotidiana y que puede enmarcar un nuevo tipo de propuestas para su análisis y estudios, ya que la habilidad de razonamiento covariacional de un individuo se puede determinar solo examinando el conjunto de comportamientos y acciones mentales exhibido mientras responde a esa tarea (Carlson y Jacobs, 2003). Por ejemplo, es muy usual que los estudiantes se hagan preguntas acerca de fenómenos cambiantes que ven a su alrededor, el nivel de lluvias, la temperatura, o el salario de sus padres son algunos de estos fenómenos que pueden llegar a ser potenciadores de 
actividades que estudien estos cambios. Este proyecto de investigación plantea los mecanismos para dar respuesta a este tipo de interrogantes desde un punto de vista científico, donde el estudiante, haciendo uso de las matemáticas, pueda ser crítico frente al análisis que haga de los cambios que está estudiando. Hasta este punto, se contaba con la motivación de crear escenarios propicios para el trabajo en equipo que ayudara a disminuir los índices de violencia y de intolerancia en el centro educativo Los Pinos y que contara con la motivación de los acudientes de los estudiantes para iniciar con el proyecto que se enfocará en la construcción de conocimiento matemático en un ambiente tecnológico.

\section{Metodología}

La idea inicial de esta propuesta define la experimentación como una práctica generadora de otras prácticas que condicionan la construcción de conocimiento. Experimentar se entiende como probar la eficacia y propiedades de una cosa, en este caso se estudiarían las características no de cosas, sino de objetos propios de la matemática escolar. Kevin Bacon afirmaba que los hombres, para conocer verdaderamente la naturaleza, no deben solo estudiarla, sino adquirir nuevos conocimientos a partir de la experimentación, y bajo estas premisas, el nuevo conocimiento matemático sería interpretado como una explicación para los fenómenos de la naturaleza. La actividad fundamental de la experimentación consiste en comparar las propiedades de los modelos con las propiedades correspondientes al mundo real (Baird, 1996).

Gracias a la experimentación, se pueden proponer problemas para los cuales los estudiantes tengan explicaciones propias, y que por medio del uso del conocimiento matemático puedan justificar, desde un punto de vista matemático, la veracidad de sus afirmaciones. Una metodología basada en esta apreciación hace necesario el diseño de laboratorios que establezcan secuencias para la comprobación de hipótesis. El laboratorio se puede interpretar como un escenario para la explicación científica y la argumentación crítica de problemas. El laboratorio es una suma estructurada de actividades de modo que planeen la formulación de hipótesis y elaboración de tesis a través de la verificación experimental de la hipótesis formulada (Galetto, 2014).

El trabajo de laboratorio tiene diferentes usos, pero principalmente podemos enmarcarlos de la siguiente manera (Hodson, 1994):

- Para motivar mediante la estimulación del interés y la diversión.

- Para intensificar, facilitar y propiciar la conceptualización de los elementos que conforman la teoría objeto de estudio.

- Para proporcionar una idea sobre el método científico y desarrollar habilidades en la planeación, organización y desarrollo del trabajo investigativo en su utilización. 
- Para desarrollar determinadas actitudes científicas como la consideración y valoración de las ideas y sugerencias de otras personas, la objetividad y la buena disposición para no emitir juicios apresurados.

Aunque estos usos son propios de prácticas experimentales en física o química, en esta propuesta son tenidas en cuenta para la elaboración de módulos de trabajo que hagan parte de un laboratorio con una visión experimental de las matemáticas. La interacción con material concreto o la construcción de dispositivos para la comprobación de resultados matemáticos que carecían de sentido son algunos de los elementos presentes en estos diseños de escenarios.

El punto inicial es la escogencia de una temática en particular de la matemática escolar y, luego de realizar un estudio epistemológico inicial, se evidencia en la mayoría de los casos una relación de dicha temática con la resolución de problemas que dieron lugar a su origen. Problemas de predicción, medición o generalización, entre otros, son algunos de los propuestos inicialmente para desarrollo del conocimiento matemático, y es en este punto donde se construyen las ideas iniciales del laboratorio y que se pretenden llevar al aula para garantizar el interés del estudiante.

Se debe tener en cuenta que, a lo largo de la historia, el hombre no ha construido sus teorías por sí solo, sino que ha continuado otros trabajos o ha interactuado con comunidades de estudio que hecho aportes para la consolidación de estas teorías. Galileo decía que un solo hombre no puede hacer ciencia (Galetto, 2014), y este aspecto se debe tener en cuenta en la concepción del laboratorio no como una metodología de trabajo individual, sino como algo que se establece bajo dinámicas de tareas grupales para la discusión de resultados y el planteamientos de preguntas que surgen en el desarrollo de estas actividades.
De esta manera, se define el laboratorio de matemáticas como una metodología de trabajo de aula que adapta ideas de la ciencias experimentales para el diseño de actividades que centren su estudio en prácticas propias de este tipo de ejercicios, (como el inferir, deducir verificar, registrar comprender, crear, predecir o crear) y no en la manipulación abstracta de contenidos matemáticos que descontextualizan este saber en la escuela de hoy.

El objetivo principal de este proyecto de investigación es encontrar los elementos teóricos y metodológicos para el diseño un laboratorio de ciencias donde se analice el uso del pensamiento covariacional en prácticas experimentales que potencien el trabajo en grupo y la convivencia con estudiantes de poblaciones vulnerable.

El proyecto define sus referentes teoricos desde la perspectiva socieepistemológica, la cual propone la unidad de análisis en las prácticas asociadas al conocimiento matematico y no en el conocimiento mismo. Desde esta misma perpectiva, se plantea la idea de posibilitar el diseño de ambientes que propicien un uso del conomiento contruyendo un nuevo significado a partir de los saberes previos de cada individuo, por lo tanto, se legitiman otros saberes distintos al escolar que acompañan la construcción de conocimiento desde lo social, dados los procesos argumentativos propios de un determinado contexto.

En esta investigación, los estudiantes presentan unos saberes previos acerca de determinados fenómenos que observan en su cotidianidad, algunas respuestas se enmarcan en una lógica propia de su entorno, pero se hace evidente la falta de respuestas a interrogantes sencillos que de una manera $u$ otra llenen sus expectativas. Por ejemplo, en una de las sesiones iniciales se les preguntó a los estudiantes cómo hacían en su casa para enfriar algun líquido. Algunas de las respuestas fueron: 
Ana: $\quad$ yo empiezo a soplar y a soplar y el chocolate se enfría.

Bernardo: depende, cuando está muy caliente, mi mamá lo coloca en un recipiente más grande.

Camila: mi mamá me enseñó a tapar el vaso con un plato y revolverlo hasta que se va enfriando.

Cada una de las respuestas evoca un conocimiento inicial para responder este interrogante, muchos de ellos responden a partir de formas y métodos heredados por costumbres familiares que demuestran las caracteristicas culturales de un saber y que ha servido para resolver algunos de los problemas tan elementales como enfriar un café. Estos saberes son el punto de partida para elaborar la metodología de trabajo con el grupo de estudiantes.

\section{Primera fase}

A partir de una convocatoria realizada en la institución en los niveles de sexto y séptimo, se entrevistó a los estudiantes que presentaban interés en la propuesta y se conformó un grupo de doce niños entre los 10 y los 12 años. Fue un denominador común en este grupo el gusto por las matematicas y el trabajo en áreas como las ciencias naturales. No se creyó conveniente utilizar ningún instrumento para medir capacidades cognitivas de los estudiantes debido a que el laboratorio que se pretende diseñar es para estudiantes en general que presenten interés por estas disciplinas y no estan supeditados a su desempeño escolar. Este punto puede general algún tipo de discusión debido a los resultados que se puedan obtener.

Posteriormente, se convocó a los padres de familia de los estudiantes para hacerlos partícipes del proyecto y explicar las fase y los alcances del mismo. Como actividad extracurricular, los padres estuviaron de acuerdo, aunque se perciben las pocas expectativas que tienen del desenvolvimiento cientifico que pueden tener sus hijos a lo largo del proyecto, es decir, dadas las pocas expectativas que tienen los padres de familia con que los estudiantes continúen sus estudios despues de su fase escolar, no es muy atrayente la idea de destacarse y proyectarse en una profesion de corte científico.

\section{Segunda fase}

Las sesiones se llevarían a cabo una vez por semana en un espacio de dos horas y en una sala de cómputo destinada por la Universidad para este proyecto. Se le suministró a cada estudiante una agenda y elementos de escritura para registrar todas las actividades y conclusiones realizadas en los laboratorios. Esta producción escrita será la base de los analisis que se hagan respecto a las argumentanciones que realizan los estudiantes en cada sesión. Se espera que, producto de las discusiones que se generen en el desarrollo de cada labarotorio, los estudiantes puedan recopilar de forma escrita o a traves de diagramas los resultados y las conclusiones que obtienen luego de la experimentación y de lo que pueden comprobar gracias 
al uso de la tecnología, en este caso con el uso del software GeoGebra. Cada laboratorio se desarrollará de la siguiente forma:

\section{Sesión de introducción}

Se comienza una discusión sobre la explicación que los estudiantes tienen acerca de determinados fenómenos físicos que ven a su alrededor. El primero de ellos gira en torno a la pregunta ¿̇cómo se enfrían los líquidos?, lo cual es algo muy cotidiano para cada uno de ellos. En esta instancia, los estudiantes comparten apreciaciones que demuestran un saber no escolar que explica este fenómeno y que en la mayoría de los casos es resultado de un conocimiento que se transmite de generación en generación a través de métodos que funcionan para cada tarea que implique la solución de un problema, en este caso el cambio de temperatura de un líquido.

Bajo la perspectiva socioepistemológica, el saber de los estudiantes y de sus familiares tiene un valor equiparable con el saber escolar, a pesar que ellos no están en condiciones de explicar por qué funciona o de optimizar sus soluciones. Uno de los objetivos de este proyecto es, a través de la ciencia, que los estudiantes estén en condiciones de dar explicaciones sobre los fenómenos físicos que pueden evidenciar y con esto poder entender el uso que tiene la ciencia y las matemáticas como recurso para comprender el funcionamiento del mundo.

En el caso particular del laboratorio, los estudiantes pueden tener varios métodos que permiten poder enfriar un líquido y se continúa con una serie de custionamientos acerca de las explicaciones que ellos le pueden dar a su método.

¿Cómo te diste cuenta que los líquidos se enfrían de esta manera?

¿Por qué crees que se enfría el líquido?
¿En qué condiciones se podría enfriar más rápido o más lento un líquido?

¿Para qué quiero que las cosas se calienten o se enfrien más rápido?

Los estudiantes no pueden responder algunas de estas preguntas, y lo que se busca es una explicación del fenómeno a partir del análisis que se puede hacer estudiando el comportamiento del mismo, para lo cual es fundamental la práctica de la medición, con la que se pueden determinar los cambios de la temperatura y registrarlos en tablas que relacionen las magnitudes. En este tipo de actividades, Sierpinska (1992) afirma que son importante factores tales como:

- Motivación: los estudiantes deben estar interesados en explicar los cambios, para así encontrar regularidades entre ellos.

- Contextos introductorios. Las funciones expresadas en forma analítica deben aparecer en primer lugar como herramientas para modelizar ciertas situaciones de la vida real o científicas.

- Contextos de desarrollo. Los métodos de interpolación se deben usar para desarrollar la noción de función.

- Comprensión de la noción de función. Los estudiantes deben ser capaces de identificar no solo aquello que cambia, sino también cómo cambia.

- Representaciones. Los estudiantes deben tener la oportunidad de adquirir cierta flexibilidad en el uso de diferentes modos de expresión y de representación.

- Metodología. La discusión en clase de las similitudes y diferencias entre las relaciones causales y las relaciones funcionales puede contribuir a la comprensión de ambas nociones. 


\section{Sesión dos}

En esta sesión, los estudiantes se enfrentan directamente con el fenómeno. No se les suministran los datos, ni tampoco se simulan a través de una aplicación tecnológica, lo que para esta propuesta se considera fundamental es que ellos sean capaces de enfrentarse a la situción y que los resultados que obtengan los consideren suyos y reales. En este caso, los estudiantes contarán con un termómetro, el cual les permitirá tomar la temperatura del líquido y poder, en intervalos de tiempo, registrar el cambio de la temperatura. Es muy importante que ellos, en la misma situación, identifiquen las magnitudes que están variando y cómo varían una con respecto a la otra. Estos cambios se interpretarán desde diferentes formas de representación, lo cual permite analizar las características y propiedades más importantes de cada comportamiento. Las notaciones pueden ser de tipo gráfico o simbólico, y para este laboratorio se tendrán en cuenta:

- Tabla de datos.

- Gráfica del comportamiento del fenómeno.

- Interpretación de las variables.

- Descripción verbal del comportamiento.

A través del tránsito entre las distintas representaciones de los comportamientos de fenómenos de la naturaleza, y en un marco de referencia físico, se caracterizará cada uno de los comportamientos, pero, según Janvier (1987), no se ha integrado el concepto de función hasta que no se es capaz de pasar de una de las representaciones a todas las demás e indico las distintas habilidades que se hacen necesarias para transitar entre las distintas representaciones de la función.

Tabla 1.

\begin{tabular}{|c|c|c|c|c|}
\hline $\begin{array}{c}\text { Descripciones } \\
\text { verbales }\end{array}$ & $\begin{array}{c}\text { Descripciones } \\
\text { verbales }\end{array}$ & Tabla de datos & $\begin{array}{c}\text { Gráficos } \\
\text { cartesianos }\end{array}$ & $\begin{array}{c}\text { Expresiones } \\
\text { algebraicas }\end{array}$ \\
\hline Tabla de Datos & Leer & Medir & $\begin{array}{c}\text { Destrezas de } \\
\text { modelización }\end{array}$ & Modelizar \\
\hline $\begin{array}{c}\text { Gráficos } \\
\text { cartesianos }\end{array}$ & Interpretar & Leer & Dibujar & Ajustar \\
\hline $\begin{array}{c}\text { Expresiones } \\
\text { algebraicas }\end{array}$ & $\begin{array}{c}\text { Reconocimientos } \\
\text { de parámetros }\end{array}$ & Calcular & Dibujar & \\
\hline
\end{tabular}

Fuente: información adaptada de Janvier, 1987.

\section{Sesión tres. Comparación y verbalización de las características}

En esta serie de sesiones se encuentra el resultado fundamental de este proyecto y es la presentación de los resultados y la comprobación de las hipótesis, esto a raíz de la importancia que tiene para el análisis poder mejorar los vínculos de convivencia y de respeto en la comunidad. La comunidad a la cual pertenecen los 
estudiantes carece de espacios donde puedan construir conocimiento a partir del trabajo en grupo y de la comparación y argumentación de ideas que busquen explicar un tipo de fenómeno, en este caso el laboratorio los lleva a que tengan un interés común y que puedan discutir validando los resultados que ellos mismos, en conjunto, encontraron y asimilaron como propios.

Los estudiantes comparten y comparan los datos que obtuvieron y buscan formas de representación que validen las hipótesis que discuten, proponen y construyen. Sus registros escritos son elementos básicos para la interpretación de las conclusiones que ellos proponen luego de una etapa de discusión, donde se generan las siguientes categorías de análisis:

- Argumentación

- Predicción

- Tolerancia

- Pensamiento crítico

El análisis de estas categorías se convertirá en la base de esta propuesta, que busca proponer un nuevo discurso escolar, más centrado en las prácticas y usando las matemáticas y la tecnología como una herramienta que ayude a la construcción de escenarios de convivencia en la concepción de una escuela más inclusiva y donde se vaya fortaleciendo el pensamiento crítico de los estudiantes a través de la explicación de fenómenos físicos cotidianos.

\section{Resultados y conclusiones}

En esta fase inicial del proyecto, se ha construido la idea del laboratorio del pensamiento variacional, un escenario que responde a las necesidades sociales de convivencia y multiculturalidad presente en la comunidad educativa del barrio Los Laches y que propone, a través de las matemáticas y la tecnología, la ampliación del discurso escolar.
Por lo anterior, los resultados del laboratorio se encaminan a poder caracterizar cada uno de los comportamientos que se describen a partir de los fenómenos físicos y que se convierten en la base del concepto de función, idea fundamental en la estructura del cálculo. En el discurso escolar, se ha privilegiado la enseñanza de la función a un tratamiento analítico que dificulta mucho la interpretación que se le puede dar a la idea de funcionalidad. Por esta razón, el laboratorio tiene como resultado inicial ser un escenario que permite la contextualización de lo funcional y establecer un grado de importancia mayor en los comportamientos físicos y no en la representación algebraica de una función.

Por otro lado, el uso del software GeoGebra ofrece a los estudiantes la posibilidad de utilizar herramientas tecnológicas en su proceso de aprendizaje, con lo cual este tipo de estudiantes presenta unas competencias tecnológicas que no los excluye del ámbito laboral donde es habitual el uso de estas herramientas. GeoGebra los ayuda a visualizar los comportamientos resultado de sus mediciones, y con esto construir argumentos para realizar sus discusiones y llegar a consensos en cada uno de los grupos de trabajo conformados. Por esta razón, la tecnología juega un papel vital en este proyecto al permitir a los estudiantes comprobar sus conjeturas y las predicciones que la fase experimental determina, con lo cual se van a establecer tres categorías que se tendrán en cuenta a partir del uso del software.

La comprobación ¿̇Qué aspectos del uso de la tecnología les permite comprobar sus hipótesis?

Otro de los factores determinantes en el análisis de los resultados del proyecto es la capacidad que tiene el grupo para exponer sus ideas y llegar a valorar los distintos saberes que tienen sus compañeros. La interpretación de esta 
información se hará a partir del estudio de cada uno de los consensos que los estudiantes hagan y de los aspectos que influyen en ellos para aceptar o compartir algún argumento. La información se obtendrá de la grabación de cada uno de los laboratorios y responderá a la siguiente categoría:

\section{Consenso: ¿ Qué los hace llegar a consensos a partir de la experimentación física?}

Los resultados de esta categoría definirán los parámetros que se tendrán en cuenta en la propuesta que se hará para pensar el discurso de la clase de matemáticas en la hipotética escuela del posconflicto, dado que es vital realizar desde las licenciaturas en matemáticas este tipo de reflexiones, producto de proyectos de investigación que involucren la construcción social de conocimiento, desligando nuestra mirada en los contenidos y potenciando el estudio de las prácticas que permiten dicha construcción.

La tercera categoría está centrada en el aporte que este proyecto puede hacer a la educación matemática como campo disciplinar y desde la perspectiva sociepistemológica el concepto de práctica social se entiende como aquello que hace hacer lo que un grupo social hace, es decir, lo que norma la actividad del ser humano; en este caso, el laboratorio del pensamiento variacional se convierte en un escenario normado por la práctica de la medición que propicia analizar lo que varía y cómo varía. Ese cambio se puede cuantificar a partir de mediciones con distintos instrumentos que propician la utilización de sistemas de medidas, que en algunos casos no conocían y que le pueden dar una mejor interpretación a partir de problemáticas de su entorno.

Medir se convierte en una práctica normativa de las predicciones que pueden llegar a realizar los estudiantes a partir de unos datos tomados inicialmente. Este escenario propicia la tercera categoría en el análisis de resultados del proyecto.

\section{Medición: ¿ Cómo la medición norma los argumentos predictivos de fenómenos físicos cotidianos de estudiantes de poblaciones vulnerables?}

En esta fase inicial del proyecto, y después de algunas sesiones de pilotaje, se cuenta con una estructura teórica del laboratorio de pensamiento variacional que en la continuación del proyecto definirá el estudio cualitativo que se hará del análisis de los resultados en este escenario escolar.

\section{Referencias}

Arrieta, J. (2003). Las prácticas de modelación como proceso de matematización en el aula. [Tesis de Doctorado]. Ciudad de México: Centro de Investigación y Estudios Avanzados del Instituto Politécnico Nacional. 
Baird, D. (1996). Experimentación: Una introduccion a la teoría de las mediciones y al diseño de experimentos. México: Prentice Hall.

Bishop, A. (1999). Enculturación matemática. Madrid: Paidós.

Buendía, G. (2004). Una socioepistemología del aspecto periódico de las funciones. [Tesis de Doctorado]. Ciudad de México: Centro de Investigación y Estudios Avanzados del Instituto Politécnico Nacional.

Carlson, M. y Jacobs, S. (2003). Razonamiento covariacional aplicado a la modelación de eventos. Revista Ema, pp. 121-156.

Cordero, F. y Martínez, J. (2001). La comprensión de la periodicidad en los contextos discreto y continuo. En G. Beitía (ed.), (s. f.), Acta Latinoamericana de Matemática Educativa (volumen 14, pp. 422-431). México: Grupo Editorial Iberoamérica.

Galetto, M. (2014). Saber experimentar. Bogotá: Magisterio.

Godino, J. y Batanero, C. (1996). Investigaciones sobre fundamentos teoricos y metodologicos de la educación matematica. En J. Godino y C. Batanero (s. f.), Investigaciones sobre fundamentos teóricos y metodológicos de la educación matemática (pp. 59-74). Grananda: Universidad de Granada.

Gonzales, R. (2005). Un modelo explicativo del interés hacia las matemáticas de las y los estudiantes de secundaria. Educación Matemática, Educación Matemática, 17 (1), 107-128.

Hodson, D. (1994). Hacia un enfoque más crítico del trabajo de laboratorio. Enseñanza de las Ciencias, 299-313.

Janvier, C. (1987). Problems of representation in the teaching and learning of mathematics. Nueva Jersey: Lawrence Erlbaum Associates.

Ministerio de Educación Nacional. (2004). Pensamiento variacional y tecnologías computacionales. Bogotá: Enlace Editores Ltda.

Miranda, A. (2009). Implementación de estrategias lúdicas en la enseñanza del algebra. Recuperado de http://investigacioncomunicativa.wikispaces.com/file/view

Sierpinska, A. (1992). The concept of function. Aspect of Epistemology and Pedagogy. En G. Harel y E. Dubinsky (s. f.), Understanding the notion of funtion (pp. 25-58). Cambridge: Mathematical Association of America.

\section{Para citar este artículo}

León, C. (2017). El pensamiento covariacional y GeoGebra: herramientas para la explicación científica de algunas realidades. Tecné, Episteme y Didaxis, TED, $42,159-171$ 